\title{
Courage, collaboration, complexity and chemotherapy safety: The view from the sharp end
}

by Esther Green, Rachel White, Karen Janes, Anthony Fields and Anthony Easty

\section{Abstract}

The Canadian oncology community was devastated by the news in August 2006 that a patient had died from an overdose of fluorouracil. Where we once thought our checks and balances ensured patient safety, we now knew they were not enough. Practice immediately began to change around the country. However, the incident report highlighted that there was much we still didn't know about safety issues in intravenous ambulatory chemotherapy safety in Canada. In response, an interdisciplinary, pan-Canadian team launched an 18-month exploratory study, resulting in a report identifying several safety issues and associated recommendations. This paper summarizes the key insights we have gathered for Canadian oncology nurses in being part of this study: that we need courage to come forward and disclose safety concerns; we should collaborate to come up with safety improvements that work for everyone; and we should strive to simplify our work at the sharp end by reducing complexity upstream and throughout the system.

\section{Background}

The Canadian oncology community was devastated by the news in August 2006 that a patient had died at the Cross Cancer Institute (CCI) in Edmonton, Alberta, from an overdose of fluorouracil. Denise Melanson's infusion pump was accidentally programmed to deliver the drug in four hours instead of four days. A few months later, the incident investigation (ISMP Canada, 2007) revealed that several other similar chemotherapy-related deaths had occurred recently

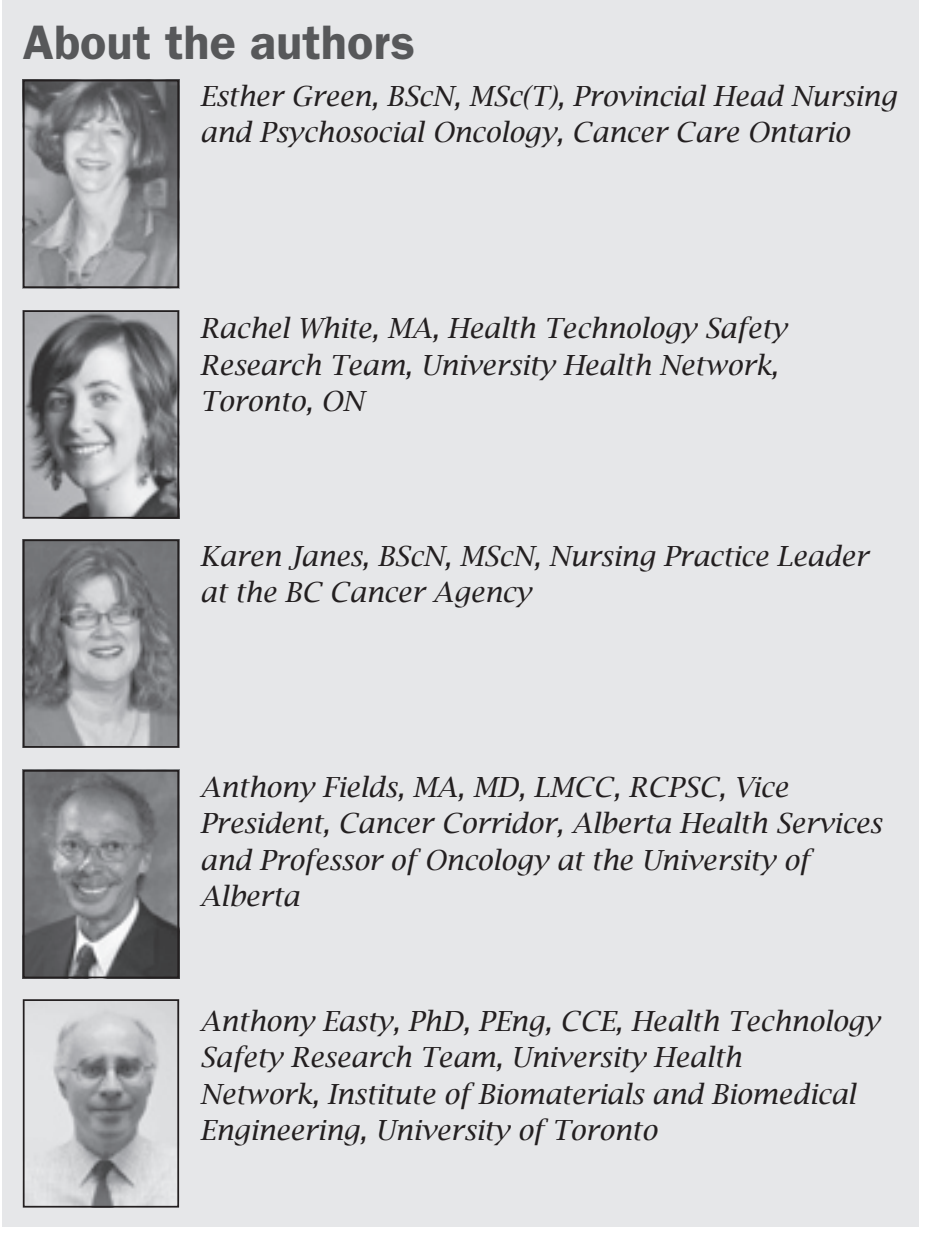

in North America, highlighting that this was not an isolated event. Where we once thought our checks and balances ensured patient safety, we now knew they were not enough.

The root cause analysis (RCA) of the fluorouracil incident by the Institute for Safe Medication Practices (ISMP) Canada identified 16 causal factors and made several associated safety recommendations. The RCA was posted on the Internet to make it widely available. Practice immediately began to change around the country in light of the recommendations. However, the report highlighted that there was much we still didn't know about safety issues in ambulatory intravenous (IV) chemotherapy in Canada. Therefore, the Canadian Association of Provincial Cancer Agencies (CAPCA) established the Systemic Therapy Safety Committee to continue work on this important topic. Under the guidance of the Committee, an inter-provincial, interdisciplinary team, submitted a grant proposal to the Canadian Patient Safety Institute's 2007 research competition. Several provinces contributed financial and in-kind resources. The study was funded and took place over a period of 18 months.

This paper summarizes the key insights that we, as members of the research team have gathered for Canadian oncology nurses by being part of this study. These relate to: courage, collaboration and complexity.

\section{The study}

This section summarizes the objectives, methods, results and recommendations from the study.

\section{Objectives}

The goals of the exploratory research study were to:

1. Identify current practices for ordering, preparing, labelling, verifying and administering ambulatory IV chemotherapy in Canada.

2. Identify additional sources of risk in a variety of ambulatory IV chemotherapy environments.

3. Recommend strategies to reduce risks.

\section{Methods}

Several methodologies were employed in the identification and prioritization of safety issues in ambulatory IV chemotherapy in Canada: a national survey of oncology care providers, field studies in six cancer treatment facilities, a proactive risk assessment based on Rasmussen's Risk Management Framework in a Dynamic Society (Rasmussen, 1997; Vicente \& Christoffersen, 2006) and an adapted Healthcare Failure Modes and Effects Analysis (South Dakota Association of Healthcare Organizations, 2009; United States Department of Veteran Affairs, 2001).

\section{Results}

A total of 331 physicians, oncology pharmacists, oncology nurses and administrators involved in cancer care from across Canada completed the survey. There was widespread awareness of the fluorouracil incident and root cause analysis report (ISMP Canada, 2007): 95.5\% of respondents were aware of the incident and $71 \%$ had reviewed the report. In total, 213 incidents were reported by the 331 respondents.

Differences between field study sites were observed in terms of: technology, models of teamwork, complexity of work processes, patient scheduling models, and role of the provincial cancer organization. Efficiency pressure was felt by staff at all sites, especially those working in pharmacy.

Data gathered using all the methodologies resulted in the identification of the following safety issues (Table 1).

\section{Recommendations}

As compared to health sciences research, in patient safety and practice research, it is rarely appropriate or feasible to use traditional quantitative evaluation methods such as randomized control trials 
in the development of recommendations. Issues and recommendations in this section are thus based on qualitative observations, enduser feedback, expert consensus, and, where they exist, best practices described in the literature.

In implementing recommendations from the report, care providers may need to be creative and make some challenging compromises to do with staffing, space and financial resources. Recommendations need to be taken in the context of local opportunity.

\section{AIPs and Access Devices}

- Elastomeric AIPs are a simple way of preventing massive flow rate errors that can occur with electronic programmable pumps, but several factors can lead to significant variance in flow rate. Improved staff and patient education is required to ensure that the pumps infuse as close to the nominal rate as possible.

- To prevent selection errors with elastomeric AIPs, which can result in significant over-infusions, the variety of models of elastomeric AIPs at each cancer treatment facility should be minimized through the simplification and standardization of protocols. Models of elastomeric AIPs with different flow rates should be stored separately from each other in pharmacy areas to prevent selection errors.

\section{Orders and Labels}

Computerized Physician Order Entry (CPOE) is often considered the best and safest approach to ordering medication. Oncology specific systems with built-in clinical decision support can reduce the risk of improper dosing and inaccurate body surface area and drug dose calculations. However, successful implementation requires significant resources and coordination, and is not an immediate possibility for many cancer care sites. For the many sites that are striving to implement pre-printed orders (PPO) as a strategy of moving away from handwritten orders, a focus of this study has been PPO design.

- For sites not using CPOE or for CPOE sites where some paper ordering still takes place, preprinted orders should be developed for all commonly used protocols. Free-form paper orders should be avoided.

- Preprinted orders should be developed according to guidelines outlined in the supplemental report Guidelines for Developing Ambulatory Chemotherapy Preprinted Orders (Healthcare Human Factors, 2010).

\begin{tabular}{|l|}
\hline Table 1. Safety issues identified and explored in research \\
\hline 1. Elastomeric ambulatory infusion pumps (AIPs) and Access \\
Devices \\
\hline 1.1 Unexplained elastomeric AIP malfunctions \\
1.2 Elastomeric AIP selection errors \\
1.3 Homecare and AIPs \\
1.4 Access devices used with elastomeric AIPs \\
\hline 2. Orders and Labels \\
\hline 2.1 Change orders \\
2.2 Pre-printed orders: reuse of forms, handwriting, usability, \\
flexibility \\
2.3 Large volume general purpose infusion pump programming \\
errors and labelling \\
2.4 Free-form orders \\
\hline 3. Pharmacy Practices \\
\hline 3.1 Organization of materials and work processes in biological \\
safety cabinets \\
3.2 No double-check of reconstitution \\
3.3 Exposure to hazardous drugs \\
\hline 4. Additional Issues \\
\hline 4.1 Patient scheduling model \\
4.2 Simplification and standardization \\
\hline
\end{tabular}

- To ensure current versions are always used, PPOs should be centrally managed and available electronically online or on the treatment centre's intranet. Orders should be printed on a per-patient basis.

- To support nurses with the task of pump programming and verification, flow rates (in the same units as the pump, e.g., $\mathrm{mL} / \mathrm{hr}$ ) should be included on pharmacy-generated chemotherapy labels and/or preprinted orders for infusions administered via large volume infusion pumps as well as AIPs.

\section{Pharmacy Practices}

- To prevent mixing errors only one chemotherapy preparation should enter the biological safety cabinet (BSC) at a time.

- To ensure the correct label is applied to its associated bag, labels and/or mixing instructions should be paired at all times with their preparation supplies and final prepared product.

- A second individual (ideally a pharmacist) should check that the correct diluent type and volume have been drawn up in the syringe for reconstitution. These checks should be in addition to any existing checks of post-constituted volumes of chemotherapy.

- Research examining the quality of mixed chemotherapy bags through techniques such as high-performance liquid chromatography is necessary to establish the mixing error rate in Canadian chemotherapy pharmacies.

\section{Other Issues}

- Simplification and standardization at the highest possible level of the healthcare system in terms of protocols, ordering tools, dosing, and workflows, will result in the greatest safety and efficiency gains. Collaboration between provincial health organizations, cancer treatment facilities and individual cancer care providers is required.

- Given the many issues stemming from the same-day model, centres should evaluate the impact of implementing a multi-day model, and where and when it is appropriate, should strive for an implementation of this scheduling approach, whereby:

- The ordering physician has access to blood test results at the time of chemotherapy ordering, and

- Treatment takes place soon after chemotherapy orders are written, but

- Treatment takes place at least one day after the orders are written so that pharmacy and nursing have time to prepare.

- Policies and procedures should be established to identify those patients for whom same-day treatment may be preferable (e.g., patients with mobility problems or those who must travel a great distance).

The full report and recommendations can be downloaded from www.capca.ca.

\section{Lessons for oncology nurses \\ Courage}

It took tremendous courage for the team at the CCI to share their

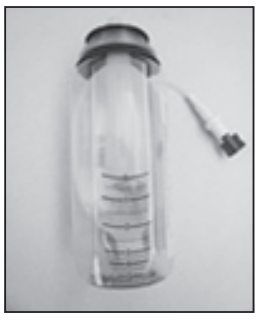

Figure 1. An elastomeric ambulatory infusion pump (Baxter Infusor, Baxter Corporation, Mississauga, ON) tragic story in 2006. Their early acknowledgment of institutional responsibility and commitment to system change was unprecedented. They took personal and professional risks by coming forward with the incident and, in doing so, alerted the oncology community to serious safety issues in systemic therapy. When the same programming error with the same drug occurred in another province a few months later, a patient's life was saved because of the lessons learned from Denise's death.

Changes to the systemic treatment system in every province and territory across Canada directly resulted from the actions of staff at the CCI. For example, there was a widespread switch away from electronic ambulatory infusion pumps (AIPs) towards mechanical elastomeric infusion pumps, to prevent massive flow 
rate errors (Figure 1). We believe these and other changes have had a direct positive impact on patient safety and may have prevented further harm to patients.

In addition to the tangible changes in practice as a result of an awareness of the fluorouracil incident, we believe the act of disclosure has had (and is still having) a significant impact on health care culture in Canada. In recent years, there has been an attempt on behalf of the safety community to shift from a person-centred approach to the management of errors towards a system approach (Reason, 2000). By acknowledging that there was no "negligence" in the fluorouracil incident, and by focusing on changing the "system," CCI has set a precedent for others to follow in disclosing errors Responses to the survey in our study illustrate this fact: 271 incidents and near misses with chemotherapy were described, and this disclosure was central to the identification and categorization of safety issues in the study.

As members of the oncology community, we encourage you to continue to follow the lead of the staff at the CCI by coming forward with adverse events and near misses. We've seen a culture shift towards "no shame, no blame" on a national level. This motto can roll off our tongues pretty easily, but there is a world of work required to make it real in the workplace. Courage is needed to share reports of our errors and near misses, and we hope you are inspired to do so in your practice, if and when the time comes.

\section{Collaboration}

This study was unique in its degree of collaboration: across jurisdictions, academic disciplines, and client-manufacturer relationships.

\section{Jurisdictional}

Geographically, five co-sponsoring provincial agencies collaborated to provide funding, as well as to volunteer field study sites. These sites universally provided a welcoming and open environment for study and collaborated with observers to highlight different approaches to practice, as well as perceived safety risks. There was also pan-Canadian representation on the project's steering committee (the CAPCA Systemic Therapy Safety Committee), which met regularly throughout the project. Further, CAPCA has been one of the key sponsors guiding the research and knowledge transfer activities, ensuring that information is shared with every possible interested party. In the nursing community in particular, CANO has been a national voice for nursing by providing support throughout the study and is now incorporating the research findings into the National Chemotherapy Administration Standards.

\section{Academic}

Collaboration also occurred between clinical disciplines and areas of expertise. Consistent with the interdisciplinary nature of how chemotherapy is administered to patients, members of the research team and steering committee represented oncology nursing, oncology pharmacy, medical oncology, radiation oncology, and

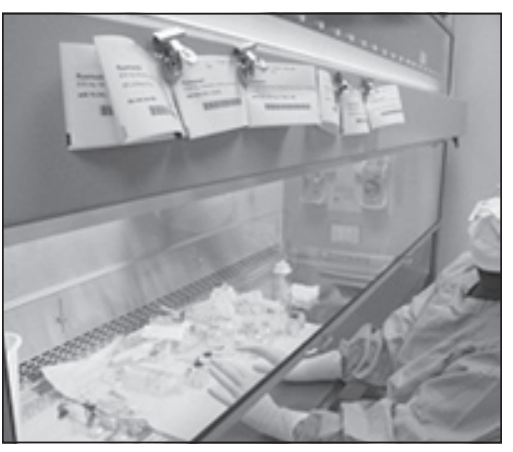

Figure 2. A pharmacy technician preparing multiple admixtures for multiple patients would not usually be involved in. For example, one of the major findings from the study related to mixing practices in pharmacy. We discovered that there is the potential for errors in mixing due to the way that the materials in the mixing area are organized, as well as the significant efficiency pressure and interruptions that are imposed on pharmacy staff (Figure 2). This finding has helped us have more understanding of the challenges our colleagues face, and the need to be cautious about the degree of time pressure we put on pharmacy. We have also realized that we are all responsible for, and susceptible to interruptions (Westbrook, Woods, Rob, Dunsmuir, \& Day, 2010). Nurses are very visible and accessible to patients and staff, and are thus very susceptible to interruptions and the associated risk of error. We are sometimes unaware of the frequency of interruptions and their impact, or we believe they are just a normal part of the job.

With guidance from the clinical team members, the research activities were primarily carried out by human factors specialists. Human factors engineering examines risk in high-performance environments based on the known skills and limitations of the human operators, with the perspective that "we cannot change the human condition, but we can change the conditions under which humans work" (Reason, 2000). These experts brought systematic tools of analysis, as well as an outside perspective on hazards in clinical practice and we believe that this engagement was integral to the success of the project. Many nurses and senior administrators are not aware of the existence of human factors engineering, or how this expertise can help clinical practice. We believe human factors training should be integral to oncology nursing education, and that human factors specialists should be central to activities such as risk assessment, technology procurement, user interface design for CPOE, and workflow design (Cafazzo et al., 2009).

As a subset of the project focused on the design of preprinted orders for chemotherapy, the team also collaborated with graphic designers to develop design guidelines and downloadable templates. This unique collaboration added to the richness of the interdisciplinary collaboration and resulted in an extremely comprehensive and easy-to-use guideline. We also believe that for some safety initiatives, the engagement of designers, along with human factors professionals, can lead to excellent results.

\section{Industry}

The third type of collaboration that took place in this study was between the research team and industry. One of the safety themes identified in the research related to elastomeric infusion pumps. Many centres had switched to these pumps to eliminate the risk of programming errors associated with electronic infusion pumps. In the survey, $96 \%$ of the elastomeric pumps were reported to be manufactured by Baxter Corporation (Baxter Corporation, n.d.). The study team, therefore, approached Baxter Canada (Mississauga, Ontario), who agreed to collaborate with us in better understanding the factors affecting the performance of these devices. We worked with them to develop new educational materials for clinicians and patients that address the problems identified in the study. We have learned from this experience that when we approach a manufacturer openly and with respect, there is great potential for collaboration and, ultimately, improvements to patient care.

We encourage you to look for opportunities to collaborate with colleagues across geographic lines and areas of expertise, as well as with technology vendors. Our experience has been that such collaborations, although sometimes logistically challenging, are extremely fruitful and, ultimately, benefit our patients.

\section{Complexity}

Systemic therapy, like much of health care, is necessarily complex. There are a vast number of systemic treatment protocols, cancer types, sites and stages. Each protocol is different in its blend of drugs, dosing, scheduling and required tests. The careful management and adjustment of protocols for individual cancer patients 


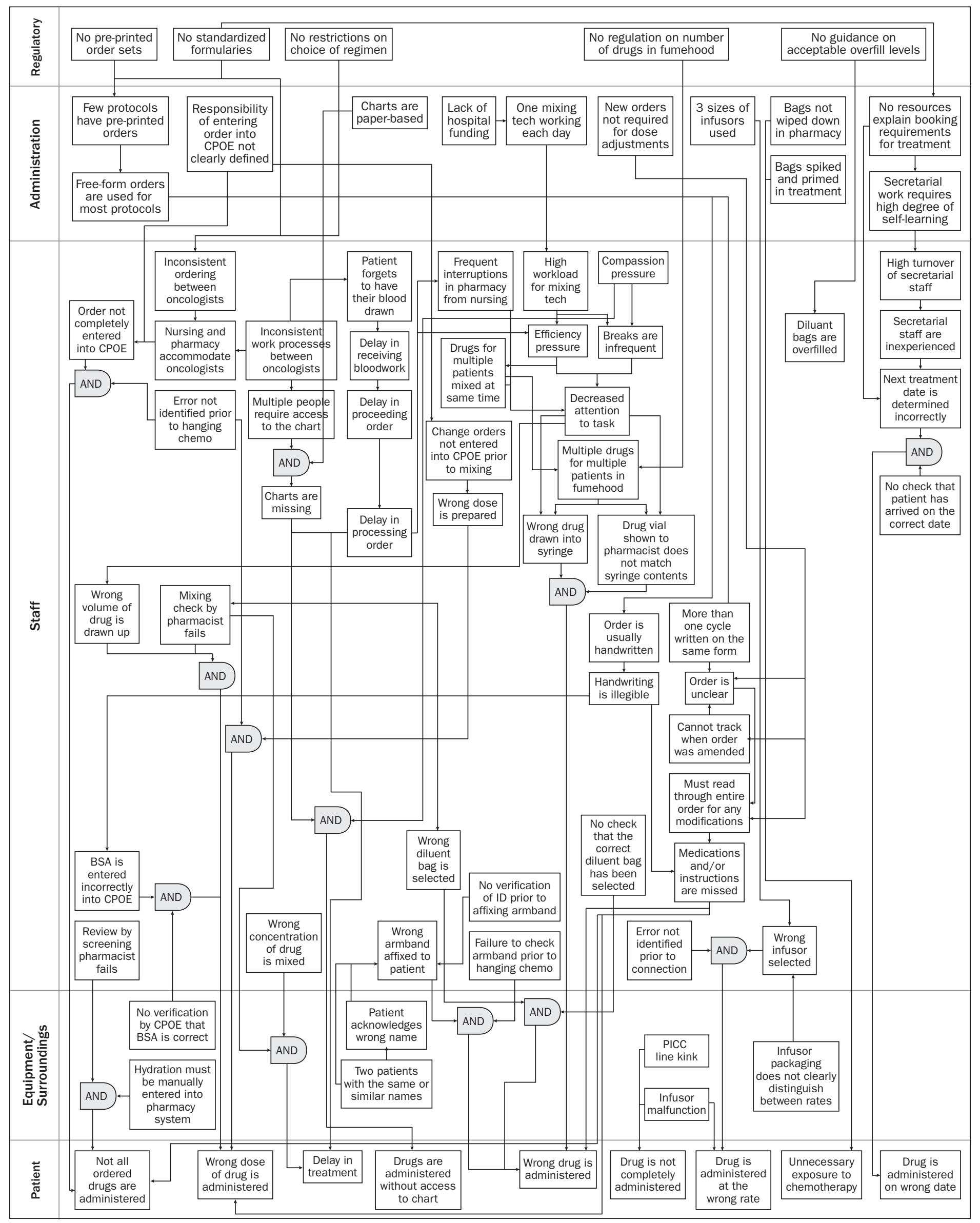

Figure 3. Complexity in chemotherapy processes 
at different stages of their treatment is critical. The systemic therapy treatment system must therefore accommodate this complexity to effectively treat patients. However, we observed that some of this complexity may not actually be required and that simplification may be possible (Figure 3).

During our week-long field study visits, we would begin by describing and mapping out the processes that all staff members follow from the time the patient is first seen by the oncologist, to when chemotherapy is ordered, prepared and administered. In some sites, we were able to grasp these front-line processes quickly, and to describe them easily in words and visual maps. However, in some sites it would take much longer to achieve this understanding and, in one case, we were never completely able to gain this understanding. While it was tempting to blame ourselves for our inability to understand the processes, when we compared practices between sites, we began to see that the issue of complexity often had to do with the degree of standardization of practices higher up in the system. For example, in one site, the oncologists chose from nearly unlimited protocols, used free-form prescription pads, and dictated the algorithms, checking practices and communication methods they desired. Each oncologist had a different practice. Therefore, the front-line staff (pharmacy and nursing) had an extremely complex system of variable processes they had to follow, and many more decisions to make. In contrast, at another site, oncologists had very limited choices of protocols for patients with specific cancer sites and stages. This standardization was at the provincial level, and the provincial agency also provided standardized ordering tools (preprinted orders). Therefore, the staff further downstream in the system had less variability to deal with, fewer decision points, less complexity and, therefore, less confusion. We believe this approach to standardization, although requiring significant coordination by senior administrators, leads to a safer system.

Another example of complexity comes directly from the fluorouracil incident. At the time the incident occurred, there were two types of electronic ambulatory infusion pumps in use in the health region. One pump had to be programmed in $\mathrm{mL}$ per hour and one in $\mathrm{mL}$ per day. The drug labels, therefore, contained both of these infusion rates. One of the immediate changes in practice following the incident was

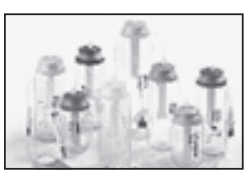

Figure 4. A variety of models of Baxter elastomeric ambulatory infusion pumps to reduce complexity of the system by standardizing the pumps used: the $\mathrm{mL}$ per day pumps were immediately removed from practice. This is an excellent example of what we can do with many aspects of systemic therapy in Canada, and we urge you to look for these types of solutions to issues at the sharp-end.

A third example of reducing complexity is an issue we identified in the study with "picking errors" of elastomeric infusion pumps. Since each model of these pumps infuses at a preset rate and there are rate options across the range
(Figure 4), it is critical that the correct device is chosen by pharmacy. Several incidents where the wrong device was filled and administered were reported in the survey and field studies. A typical safety recommendation for reducing this type of error would be to suggest policies that mandate independent double checks of these devices or to require different models of pumps to be stored in different areas of the pharmacy. Although these are important policies, a more effective strategy for preventing this type of error is to standardize practice to reduce the number of pump models that are used in a given centre. If fewer pump models are stored in a pharmacy, fewer picking errors will be possible.

We encourage you to look at the sharp end issues in your workplace and ask yourselves if they are the result of unnecessary complexity. We need to work together to decide on standards of practice that make for fewer decision points, and less confusion.

\section{Conclusions}

The tragedy of one life lost in August 2006 was a powerful stimulus, triggering a series of important actions. Widespread practice changes took place around the country, and we believe other lives have been or will be saved, as a result.

Colleagues in human factors remind us that we will always live in an imperfect world, and that, as humans, we will always be fallible. If an error potentially can happen, it eventually will happen. Removing these potential errors before they occur is a great step toward improved safety. Remember that we cannot change the human condition but, as nurses and health care administrators, we can change the conditions under which we work. We can have courage in coming forward and disclosing safety concerns, we can collaborate to come up with safety improvements that work for everyone, and we can strive to simplify things at the sharp end by reducing complexity upstream and throughout the system.

\section{Acknowledgements}

The research team included members from many organizations and disciplines: Anthony Easty, Dr. Anthony Fields, Rachel White, Esther Green, Karen Janes, Andrea Cassano-Piche, Jennifer Jeon, Debbie Chan, Roxanne Dobish, Dr. Dhali Dhaliwal, Dr. Eshwar Kumar, Venetia Bourrier, Sylvia Hyland, Dr. Brent Schacter, Dr. Maureen Trudeau, Dr. Yoo-Joung Koo and Kim Vicente. Members of the CAPCA Systemic Therapy Safety Committee included: Susan Walisser, Dr. Susan O'Reilly, Denise Budz, Larry Broadfield, Heather MacKenzie, Roxanne Rodgers-Harding, Scott Edwards, Nancy Bestic, Jessica Peters, Melany Leonard, Heather Logan, and Jillian Hardy The authors and the members of the research team wish to thank the nurses from across Canada who participated in the study, and CANO/ACIO that supported the study. Finally, the authors thank and acknowledge the courageous team at the Cross Cancer Institute and the family of Denise Melanson.

\section{References}

Baxter Corporation. (n.d.). INFUSOR Portable Elastomeric Infusion System. Retrieved from http://www.ecomm.baxter.com/ecatalog/ browseCatalog.do?lid=10001\&hid=10001\&cid $=10016 \&$ key $=771 \mathrm{de} 8$ 597ad1a2d53ff3a83bf1fe4e

Cafazzo, J.A., Trbovich, P., Cassano-Piche, A., Chagpar, A., Rossos, P.G., Vicente, K.J., \& Easty, A.C. (2009). Human factors perspectives on a systemic approach to ensuring a safer medication delivery process. Healthcare Quarterly, 12(Sp), 70-74.

Healthcare Human Factors. (2010). Recommendations for improving ambulatory chemotherapy preprinted orders, 2010. Toronto, ON: Author.

Institute for Safe Medication Practices Canada (ISMP). (2007). Fluorouracil root cause analysis: Follow up 2007. Retrieved from http://www.ismp-canada.org/download/safetyBulletins/ SMPCSB2007-04Fluouracil.pdf

Rasmussen, J. (1997). Risk management in a dynamic society: A modeling problem. Safety Science, 27(2/3), 183-213.

Reason J. (2000). Human error: Models and management. BMJ, 320(7237), 768-70.

South Dakota Association of Healthcare Organizations. (2009). FMEA Scoring Scale, 2009. Sioux Falls, SD: Author.

United States Department of Veteran Affairs. (2001). The basics of healthcare failure mode and effect analysis. Ann Arbor, MI: Veteran Affairs National Center for Patient Safety.

Vicente, K., \& Christoffersen, K. (2006). The Walkerton E. coli outbreak: A test of Rasmussen's framework for risk management in a dynamic society. Theoretical Issues in Ergonomics Science, 7(2), 93-112.

Westbrook, J.I., Woods, A., Rob, M.I., Dunsmuir, W.T., \& Day, R.O. (2010). Association of interruptions with an increased risk and severity of medication administration errors. Arch Intern Med, 170(8), 683-90. 